\title{
Symmetrization of the Classical "Attack-defense” Model
}

\section{Pavel Yuryevich Kabankov ${ }^{1}$, Alexander Gennadevich Perevozchikov ${ }^{1}$, Valery Yuryevich Reshetov ${ }^{2}$, Igor Evgenievich Yanochkin ${ }^{1}$}

${ }^{1}$ Department of System Design, JSC NPO RusBITekh-Tver, Tver, Russia

${ }^{2}$ Faculty of Computational Mathematics and Cybernetics, Lomonosov Moscow State University, Moscow, Russia

\section{Email address:}

kpy@rusbitech.ru (P. Y. Kabankov), pere501@yandex.ru (A. G. Perevozchikov), kadry@cs.msu.ru (V. Y. Reshetov), i-yanochkin@yandex.ru (I. E. Yanochkin)

\section{To cite this article:}

Pavel Yuryevich Kabankov, Alexander Gennadevich Perevozchikov, Valery Yuryevich Reshetov, Igor Evgenievich Yanochkin. Symmetrization of the Classical "Attack-defense" Model. Science Journal of Applied Mathematics and Statistics.

Vol. 8, No. 1, 2020, pp. 1-10. doi: 10.11648/j.sjams.20200801.11

Received: December 7, 2019; Accepted: December 18, 2019; Published: January 7, 2020

\begin{abstract}
The article considers Germeyer's "doubled" classic "attack-defense" game, which is symmetrical for the participants in the sense that in one game each participant is an "attack" party and in the other game each participant is a "defense" party. This corresponds to the logic of bilateral active-passive operations, when the parties simultaneously conduct defensive-offensive operations against each other. The mathematical expectation of the number of destroyed enemy means is taken as criteria for the effectiveness of the parties, which should be maximized implicitly. Thus, both sides are placed in a "defense" position. Under otherwise equal conditions, the parties strive to minimize shares aimed at defense, guided by a strategy of reasonable sufficiency of defense. The authors study Pareto-dominated equilibria depending on the initial ratio of the parties forces and, in particular, the extreme points of Pareto sets. Formulas are obtained for such equilibria depending on the parties' balance of forces, which allows us to build a dynamic expansion of the model in the future. The main research method is the parametrization of Nash's equilibria. The parameterization of the equilibria shows that they fill the two-dimensional subregion of a unit square with a boundary. Therefore, for its narrowing, it makes sense to distinguish from it the Pareto-non-dominated part of the boundary and its extreme points. The latter provide an opportunity to assess the maximum share of the strike means of the parties, which they can afford to allocate without prejudice to the defense. It is shown that these fractions represent piecewise continuous functions of the initial ratio of the parties' forces and explicit expressions for them are obtained. A numerical example of the construction of the Paretonon-dominated part of the boundary and its extreme points is given.
\end{abstract}

Keywords: Germeyer's Classical “Attack-Defense" Game, Multi-Turn Generalization, Best Guaranteed Result of Defense, Game's "Doubling", Equilibrium Strategies Parameterization,

Pareto-Minimal Set of Equilibria, Pareto-Minimal set Extreme Points

\section{Introduction}

The work is based on the results from [1-2] and is a further development of the constructions in [3-4]. Germeyer's classical "attack-defense" model was defined and studied in [5]. It is a modification of the Gross' model [6]. In the military models points are usually interpreted as directions and characterize the spatial distribution of defense resources across the width. However, in reality there is also a spatial distribution of defense resources in depth, characterized by the number of levels of defense lines in this direction.

The simplest model was proposed in [5], taking into account the defense's lines. A game model that generalized the Gross and Germeyer models was studied in [7]. In this model a constructive description of the set of all optimal mixed attack strategies was obtained. The Gross's model with the opposite interests of the parties was studied in [8], and dynamic extensions of the model were studied in $[9,10]$. a direct generalization of the attack-defense game on networks describing the topology of the paths leading to defended objects was proposed in [11].

The further generalization of the classical "attack-defense" game may consist in its symmetrization, which leads in the general case to problems of finding equilibria with concave 
criteria, that can be reduced to solving a system of inclusions [12]. The parameterization of the equilibria shows that they fill the two-dimensional subregion of a unit square with a boundary. Therefore, for its narrowing, it makes sense to distinguish from it the Pareto-non-dominated part of the boundary and its extreme points. The latter provide an opportunity to assess the maximum share of the strike means of the parties, which they can afford to allocate without prejudice to the defense. It is shown that these fractions represent piecewise continuous functions of the initial ratio of the forces of the parties and explicit expressions for them are obtained, which can be used in the dynamic expansion of the model according to the scheme [13].

\section{The Simplest Multi-Line Generalization of the Model}

The simplest model, taking into account the defense's lines, consists in modifying the classical model [5], in which the function of attack's winning has the form [1]:

$$
f(x, y)=\sum_{i=1}^{n} \max \left[q_{i}^{T_{i}} x_{i} ; x_{i}-p_{i} r_{i} y_{i}\right]
$$

and the vectors $x, y$ belong to the sets

$$
M=\left\{x \in E_{+}^{n} \mid \sum_{i=1}^{n} x_{i}=X\right\}, N=\left\{y \in E_{+}^{n} \mid \sum_{i=1}^{n} y_{i}=Y\right\},
$$

where $T_{i}$ is the number of defense lines in the $i-$ th direction $i=1, \ldots, n, r_{i}$ - the maximum number of actions that one unit of defense can produce, $p_{i}$ - the probability of hitting one enemy attack means with one effect in the $i-$ th direction, which is assumed to be independent from the number $t=1, \ldots, T_{i}$ of the defense line, $q_{i}=1-p_{i}$ - the corresponding probability of non-defeat, $X$ and $Y$ is the total number of attack and defense means, which are considered homogeneous and infinitely divisible, $x_{i}$ and $y_{i}$ - the number of attack and defense means in the $i-$ th direction. In particular, formally, when $p_{i}=1$ we obtain the Germeier's classical model [5].

The content of the model is interpreted as follows: the attack party strive to maximize the total amount of means that break through, distributing its means in $n$ directions. Defense party, on the contrary, strive to minimize the number of means that break through by distributing its means in $n$ directions. In each direction, the attack party must overcome the layered defense of the enemy. The result of a combat collision at one defense line is given by a function $x_{i}^{\prime}=\max \left[q_{i} x_{i} ; x_{i}-p_{i} r_{i} y_{i}\right]$ that is the result of the OsipovLanchester's discrete one-step model of dynamics of average. It is obtained as follows: the number of attack means that have received an impact at a given line is $n_{i}=\min \left[x_{i} ; r_{i} y_{i}\right]$ providing that exactly one defense means effect one attack means. The mathematical amount of attack's losses will be $m_{i}=p_{i} n_{i}$. As a result, the mathematical expectation of the number of means that break through will be $x_{i}^{\prime}=x_{i}-m_{i}=\max \left[q_{i} x_{i} ; x_{i}-p_{i} r_{i} y_{i}\right]$. The final formula for the mathematical expectation of attack means overcoming all $T_{i}$ defense's lines will be $x_{i}^{\prime}=\max \left[q_{i}^{T_{i}} x_{i} ; x_{i}-p_{i} r_{i} u_{i}\right]$ taking into account the optimization for the defense party of the distribution of its means $y_{i}$ at the lines and derived in [1].

Using the convexity of the function $f(x, y)$, it was proved for this antagonistic game (see [1]) that the best guaranteed result (BGR) of defense

$$
\left.v=\max \left(X \max _{i=1,2, \ldots, n} q_{i}^{T_{i}} ; X-Y\left(\sum_{i=1}^{n} \frac{1}{p_{i} r_{i}}\right)^{-1}\right)\right)
$$

will be the value of the game and the minimax defense strategy is optimal. In this case, the optimal attack strategy is a mixed strategy, consisting in concentrating all forces in one direction in accordance with the optimal probability distribution, which can be obtained by the formulas given in [1].

Let's denote for brevity

$$
Q=\max _{i=1,2, \ldots, n} q_{i}^{T_{i}}, P=1-Q, t=\left(\sum_{i=1}^{n} \frac{1}{p_{i} r_{i}}\right)^{-1}
$$

\section{Model's Symmetrization}

Let's suppose that strike forces and defense forces take part in a game of two sides $B, A$. The proportion of strike forces and defense forces is $\sigma^{B}, \sigma^{A}$ from the total number of parties' means $Y^{B}, Y^{A}$. Then the losses of the opposite sides will be according to formula (3), taking into account the accepted notation (4)

$$
\begin{aligned}
& F\left(\sigma^{B}, \sigma^{A}\right)=Y^{A}\left(1-\sigma^{A}\right)- \\
& -\max \left\{Q^{B} Y^{A}\left(1-\sigma^{A}\right), Y^{A}\left(1-\sigma^{A}\right)-t^{B} Y^{B} \sigma^{B}\right\}= \\
& =\min \left(P^{B} Y^{A}\left(1-\sigma^{A}\right), t^{B} Y^{B} \sigma^{B}\right),
\end{aligned}
$$

and

$$
\begin{aligned}
& G\left(\sigma^{B}, \sigma^{A}\right)=Y^{B}\left(1-\sigma^{B}\right)- \\
& -\max \left\{Q^{A} Y^{B}\left(1-\sigma^{B}\right), Y^{B}\left(1-\sigma^{B}\right)-t^{A} Y^{A} \sigma^{A}\right\}= \\
& =\min \left(P^{A} Y^{B}\left(1-\sigma^{B}\right), t^{A} Y^{A} \sigma^{A}\right) .
\end{aligned}
$$

The payment functions of the parties are continuous and concave; therefore, by virtue of Theorem 8 in [12, p. 90], there are situations of parties' equilibrium that satisfying the inclusions 


$$
\sigma_{0}^{B} \in \Sigma\left(\sigma_{0}^{A}\right), \sigma_{0}^{A} \in \Xi\left(\sigma_{0}^{B}\right)
$$

where

$$
\begin{aligned}
& \Sigma\left(\sigma^{A}\right)=\operatorname{Arg} \max _{\sigma^{B} \in[0,1]} F\left(\sigma^{B}, \sigma^{A}\right), \\
& \Xi\left(\sigma^{B}\right)=\operatorname{Arg} \max _{\sigma^{A} \in[0,1]} G\left(\sigma^{B}, \sigma^{A}\right)-
\end{aligned}
$$

sets of the parties' best answers. In this case, it can be easy to find explicitly.

$$
\begin{aligned}
& \Sigma\left(\sigma^{A}\right)=\left[\min \left(\frac{P^{B} Y^{A}\left(1-\sigma^{A}\right)}{t^{B} Y^{B}}, 1\right) ; 1\right], \\
& \Xi\left(\sigma^{B}\right)=\left[\min \left(\frac{P^{A} Y^{B}\left(1-\sigma^{B}\right)}{t^{B} Y^{A}}, 1\right) ; 1\right] .
\end{aligned}
$$

Or

$$
\Sigma\left(\sigma^{A}\right)=\left\{\begin{array}{l}
\{1\}, 0 \leq \sigma^{A}<1-\frac{t^{B} Y^{B}}{P^{B} Y^{A}}, \\
{\left[\frac{P^{B} Y^{A}\left(1-\sigma^{A}\right)}{t^{B} Y^{B}}, 1\right], 1-\frac{t^{B} Y^{B}}{P^{B} Y^{A}} \leq \sigma^{A} \leq 1,}
\end{array}\right.
$$

and

$$
\Xi\left(\sigma^{B}\right)=\left\{\begin{array}{l}
\{1\}, 0 \leq \sigma^{B}<1-\frac{t^{A} Y^{A}}{P^{A} Y^{B}}, \\
{\left[\frac{P^{A} Y^{B}\left(1-\sigma^{B}\right)}{t^{A} Y^{A}}, 1\right], 1-\frac{t^{A} Y^{A}}{P^{A} Y^{B}} \leq \sigma^{B} \leq 1 .}
\end{array}\right.
$$

\section{Parameterization of Equilibria}

Parameterization of all Nash's equilibria can be obtained by choosing specific points from segments (9) in inclusion (7),

$$
\begin{aligned}
& \sigma^{B}=(1-\lambda) \min \left(\frac{P^{B} Y^{A}\left(1-\sigma^{A}\right)}{t^{B} Y^{B}}, 1\right)+\lambda, \\
& \sigma^{A}=(1-\mu) \min \left(\frac{P^{A} Y^{B}\left(1-\sigma^{B}\right)}{t^{A} Y^{A}}, 1\right)+\mu,
\end{aligned}
$$

where

$$
\lambda, \mu \in[0,1]
$$

- some parameters.

Equilibria correspond to points satisfying the conditions

$$
0 \leq \sigma^{A} \leq 1,0 \leq \sigma^{B} \leq 1 .
$$

Let's consider a subset $\Omega \subseteq[0,1] \times[0,1]$ of such pairs $(\lambda, \mu)$ under the conditions (12) - (14) are compatible. The mathematical expectation of the number of destroyed enemy means is taken as criteria for the effectiveness of the parties, which should be implicitly. Thus, both sides are placed in a "defense" position. The strategy of each side is the share of forces directed to defense. Let's suppose that under otherwise equal conditions, the parties adhere to a strategy of reasonable defense adequacy. The desire to minimize the shares $\sigma^{B}, \sigma^{A}$ allocated for defense can be formalized by considering its one-dimensional subset $\Omega^{\prime}$ of Paretominimal points $(\lambda, \mu) \in \Omega$ and its images $\left(\sigma^{B}, \sigma^{A}\right)$. Therefore, further the Pareto-dominated equilibria (12) - (14) are studied depending on the initial ratio of the parties forces and, in particular, the extreme points of the Pareto sets.

For the attack party, which has a noticeable excess of the balance of forces in its favor, it makes sense to choose an equilibrium corresponding to the minimum value of the share allocated to cover. The minimization of the criterion $\sigma^{B}(\lambda, \mu)$ on the set $\Omega^{\prime}$ of Pareto-non-dominated equilibria gave such a solution. We will call it a solution of the minimum sufficiency of defense for the attack party. Note that in this case $\sigma^{A}(\lambda, \mu)$ reaches a maximum on the set $\Omega^{\prime}$ due to the Pareto's non-dominance.

Let's suppose that $Y^{B}, Y^{A}>0$. Denote the ratio of the parties forces the from the point of view $B$ by $x=Y^{B} / Y^{A}$. Assume, following [14], that the conditions are correct

$$
P^{B} / t^{B}<1, P^{A} / t^{A}<1
$$

Let's consider the various cases that arise when the minima are revealed in (12). Depending on whether the minimum in the first and second equations in (12) is equal to the left (L) or right $(\mathrm{R})$ expression, we'll consider the LL, LR, RL, and RR options.

\subsection{The Main Case LL}

Let's suppose that the minima in (12) are reached on the first component. Then (12), (14) are equivalent to the system

$$
\sigma^{B}=\frac{(1-\lambda) P^{B} Y^{A}\left(1-\sigma^{A}\right)}{t^{B} Y^{B}}+\lambda, \sigma^{A}=\frac{(1-\mu) P^{A} Y^{B}\left(1-\sigma^{B}\right)}{t^{A} Y^{A}}+\mu
$$

with conditions

$$
1-\frac{t^{B} Y^{B}}{P^{B} Y^{A}} \leq \sigma^{A} \leq 1,1-\frac{t^{A} Y^{A}}{P^{A} Y^{B}} \leq \sigma^{B} \leq 1,
$$

Remark 1. The point $\left(\sigma^{B}, \sigma^{B}\right)=(1,1)$ is always the equilibrium and corresponds to the point $(\lambda, \mu)=(1,1) \in \Omega$. Therefore, it is interesting to obtain conditions under which $(\lambda, \mu)=(0,0) \in \Omega$, i.e. $\Omega=[0,1] \times[0,1]$ and $\Omega^{\prime}=\{(0,0)\}$ consists of a single point.

System $(16),(17)$, when $(\lambda, \mu)=(0,0)$ taking into account the accepted notation, has the form of conditions

It is easy to verify that the left inequalities in (19) to be verified, taking into account (18), are collectively equivalent to the right. Therefore, system (19) is equivalent to the conditions 


$$
\sigma^{B}=\frac{P^{B}}{t^{B}} \frac{1-\sigma^{A}}{x}, \sigma^{A}=\frac{P^{A}}{t^{A}} x\left(1-\sigma^{B}\right),
$$

and

$$
1-\frac{t^{B}}{P^{B}} x \leq \sigma^{A} \leq 1,1-\frac{t^{A}}{P^{A}} \frac{1}{x} \leq \sigma^{B} \leq 1 .
$$

It is easy to verify that the left inequalities in (19) that were subjects to verification are collectively equivalent to the right inequalities taking into account (18). Therefore the system (19) is equivalent to the conditions

$$
1-\frac{t^{B}}{P^{B}} x \leq \sigma^{A}, 1-\frac{t^{A}}{P^{A}} \frac{1}{x} \leq \sigma^{B},
$$

and

$$
\sigma^{A}, \sigma^{B} \geq 0
$$

\subsubsection{Case When the Left Sides in (20) Are Nonpositive}

We'll First Consider the Case When the Left Sides in (20) Are Nonpositive, then (21) Implies (20) and the Solution to the System (18), (20) - (21) Relatively ${ }^{x}$ Is the Segment

$$
\frac{P^{B}}{t^{B}} \leq x \leq \frac{t^{A}}{P^{A}} .
$$

Note that due to inequalities (15), the right side of (22) is larger than the left one.

Let's solve system (18) with respect to $\sigma^{B}$ :

$$
\sigma^{B}=\frac{P^{B}}{t^{B}} \frac{\left(1-\frac{P^{A}}{t^{A}} x\right)}{x\left(1-\frac{P^{B}}{t^{B}} \cdot \frac{P^{A}}{t^{A}}\right)} .
$$

Then $\sigma^{B} \geq 0$ by virtue of (15), (22). It follows from (20) that $\sigma^{B}, \sigma^{A} \leq 1$, whence it follows that

$$
\sigma^{A}=\frac{P^{A}}{t^{A}} x\left(1-\sigma^{B}\right) \geq 0 .
$$

Thus $(\lambda, \mu)=(0,0) \in \Omega$ in case $(22)$.

\subsubsection{Case When $(\lambda, \mu)=(0,0) \notin \Omega$}

Let's Now

$$
x>\frac{t^{A}}{P^{A}}>\frac{P^{B}}{t^{B}} .
$$

Then condition (20) is equivalent to the right inequality

$$
1-\frac{t^{A}}{P^{A}} \frac{1}{x} \leq \sigma^{B}
$$

which should be solved in conjunction with (21). However, the latter is false due to the fact that $\sigma^{B}<0$ by virtue of (23). Thus, under condition (25), the point $(\lambda, \mu)=(0,0) \notin \Omega$.

\subsubsection{Symmetric Case Let's Now}

$$
x<\frac{P^{B}}{t^{B}}<\frac{t^{A}}{P^{A}} .
$$

Remark 2. Case (27) is symmetric (25); therefore, it can not be considered separately. In fact, condition (22) is invariant with respect to the replacement $B$ by $A$ and $x$ by $\frac{1}{x}$, whereby case (27) goes over into (25).

Thus, the following theorem is valid.

Theorem 1. A necessary and sufficient condition that the set $\Omega^{\prime}$ consist of a single point $\{(0,0)\}$ is condition (22).

Remark 3. The only solution that appears in Theorem 1 is stable provided that the latter adheres to a strategy of reasonable sufficiency of defense, i.e. it strive to minimize the share of its funds allocated for defense when reaching the maximum of its criterion, as the second parties in the Stackelberg equilibrium (see [12], p. 122). Therefore, it can be called the Stackelberg symmetrized equilibrium.

Remark 4. It seems unnatural to play along with your opponent in the war game using the strategies of the second players in the Stackelberg equilibria, but this contradiction is easily eliminated by moving to the vector criteria of the parties $\left(F\left(\sigma^{B}, \sigma^{A}\right), 1-\sigma^{B}\right)$ and $\left(G\left(\sigma^{B}, \sigma^{A}\right), 1-\sigma^{A}\right)$, accordingly, to the lexicographic equilibrium, which is defined similarly to the classical Nash's equilibrium using the concept of the lexicographic maximum of the vector criterion. This equilibrium will also be equivalent to a system of inclusions of parties strategies to point-to-multiple mappings that implement the lexicographic maximum of each party, as multivalued functions of the strategy of the opposite side. After that, each party "plays along" with itself according to the second criterion $1-\sigma^{B}$ and $1-\sigma^{A}$ accordingly. We prefer to stay in this article within the framework of the formalism of Stackelberg symmetrized equilibrium so as not to complicate the proof of the main results, since the second criteria are equivalent to the criteria $G\left(\sigma^{B}, \sigma^{A}\right)$ and $F\left(\sigma^{B}, \sigma^{A}\right)$ in the sense of monotonicity by $1-\sigma^{B}$ and $1-\sigma^{A}$, accordingly, by the definition of criteria (5), (6).

\subsubsection{Researching the Obtained Solution}

The general solution of system (16) relatively $\sigma^{B}$ has the form

$$
\sigma^{B}=1-\frac{x-\frac{P^{B}}{t^{B}}-\lambda}{x g(\lambda, \mu)}
$$

where indicated for brevity

$$
g(\lambda, \mu)=1-\frac{P^{B}}{t^{B}} \cdot \frac{P^{A}}{t^{A}}(1-\lambda)(1-\mu)>0 .
$$


In order to $\sigma^{B}<1$ it is need to

$$
x-\frac{P^{B}}{t^{B}}-\lambda>0 .
$$

Derivatives $\sigma^{B}$ of $\lambda, \mu$ has the form

$\sigma_{\lambda}^{B}=\frac{g(\lambda, \mu)+\left(x-\frac{P^{B}}{t^{B}}-\lambda\right) g_{\lambda}(\lambda, \mu)}{x g^{2}(\lambda, \mu)}, \sigma_{\mu}^{B}=\frac{\left(x-\frac{P^{B}}{t^{B}}-\lambda\right) g_{\mu}(\lambda, \mu)}{x g^{2}(\lambda, \mu)}$

and are positive under condition (30) by virtue of the inequalities

$\left.\left.g_{\lambda}(\lambda, \mu)=\frac{P^{B}}{t^{B}} \cdot \frac{P^{A}}{t^{A}}(1-\mu)\right)>0, g_{\mu}(\lambda, \mu)=\frac{P^{B}}{t^{B}} \cdot \frac{P^{A}}{t^{A}}(1-\lambda)\right)>0$,

that correct at $\lambda<1, \mu<1$.

\subsection{Additional Case LL}

We'll solve system (16), (17) in the general case when condition (22) is not satisfied, i.e.

$$
\left.x \notin\left[\frac{p^{B}}{t^{B}}, \frac{t^{A}}{p^{A}}\right] .\right)
$$

Let's suppose for definiteness, that case (25) holds, i.e. the side $B$ has an advantage in the balance of forces. Case (27) is symmetric by virtue of Remark 5; therefore, it can not be considered separately.

The solution of system (16) - (17) relatively $\sigma^{B}$ has the form (31)

$$
\begin{gathered}
\sigma^{B}=\frac{\frac{P^{B}}{t^{B}}-\frac{P^{B}}{t^{B}} \cdot \frac{P^{A}}{t^{A}} x(1-\lambda)(1-\mu)+\lambda}{x\left(1-\frac{P^{B}}{t^{B}} \cdot \frac{P^{A}}{t^{A}}(1-\lambda)(1-\mu)\right)} . \\
x \leq\left(\frac{\frac{P^{B}}{t^{B}}+\lambda}{1-\frac{P^{B}}{t^{B}} \cdot \frac{P^{A}}{t^{A}}(1-\lambda)(1-\mu)}+\frac{t^{A}}{P^{A}}\right)\left(1-\frac{P^{B}}{t^{B}} \cdot \frac{P^{A}}{t^{A}}(1-\lambda)(1-\mu)\right)= \\
=\frac{P^{B}}{t^{B}}+\lambda+\frac{t^{A}}{P^{A}}\left(1-\frac{P^{B}}{t^{B}} \cdot \frac{P^{A}}{t^{A}}(1-\lambda)(1-\mu)\right)=\frac{P^{B}}{t^{B}}+\lambda+\frac{t^{A}}{P^{A}}-\frac{P^{B}}{t^{B}}(1-\lambda)(1-\mu) .
\end{gathered}
$$

Inequality (17) is equivalent to the right inequality

$$
1-\frac{t^{A}}{P^{A}} \frac{1}{x} \leq \sigma^{B}
$$

which should be solved in conjunction with the condition

$$
\sigma^{B} \leq 1
$$

In fact, it follows from (14) and (33) that $\sigma^{B} \geq 0$, and the condition is equivalent to the condition

$$
\frac{P^{A} Y^{B}\left(1-\sigma^{B}\right)}{t^{A} Y^{A}} \leq 1
$$

which is a consequence of (33).

Condition (34) is equivalent to the inequality

$$
\frac{P^{B}}{t^{B}}+\lambda \leq x
$$

The condition (33) is remains, which, taking into account (32), takes the form

$$
\frac{\frac{P^{B}}{t^{B}}-\frac{P^{B}}{t^{B}} \cdot \frac{P^{A}}{t^{A}} x(1-\lambda)(1-\mu)+\lambda}{x\left(1-\frac{P^{B}}{t^{B}} \cdot \frac{P^{A}}{t^{A}}(1-\lambda)(1-\mu)\right)} \geq 1-\frac{t^{A}}{P^{A}} \frac{1}{x} .
$$

The latter is equivalent to the inequality

$$
\frac{1}{x}\left(\frac{\frac{P^{B}}{t^{B}}+\lambda}{1-\frac{P^{B}}{t^{B}} \cdot \frac{P^{A}}{t^{A}}(1-\lambda)(1-\mu)}+\frac{t^{A}}{P^{A}}\right) \geq 1+\frac{\frac{P^{B}}{t^{B}} \cdot \frac{P^{A}}{t^{A}}(1-\lambda)(1-\mu)}{1-\frac{P^{B}}{t^{B}} \cdot \frac{P^{A}}{t^{A}}(1-\lambda)(1-\mu)} .
$$

In the view of (35), we finally obtain the condition

$$
\frac{P^{B}}{t^{B}}+\lambda \leq x \leq \frac{P^{B}}{t^{B}}+\lambda+\frac{t^{A}}{P^{A}}-\frac{P^{B}}{t^{B}}(1-\lambda)(1-\mu) .
$$

Thus, the set $\Omega$ of admissible $(\lambda, \mu)$ in case (25) is determined by conditions (13), (36).

By virtue of (32) $\sigma^{B}(\lambda, \mu)$ it can be represented in the form (31)

$$
\sigma^{B}=1-\frac{x-\frac{P^{B}}{t^{B}}-\lambda}{x\left(1-\frac{P^{B}}{t^{B}} \cdot \frac{P^{A}}{t^{A}}(1-\lambda)(1-\mu)\right)} .
$$

Let's note that by virtue of condition (36), the fraction on the right side is a non-negative quantity.

Theorem 2. Let's suppose that condition (25) is satisfied. Then the necessary condition for the minimality of the 
criterion $\sigma^{B}(\lambda, \mu)$ on the set $\Omega$ is the conditions $\lambda=0$ or $\mu=1$.

Evidence. If for some $(\lambda, \mu) \in \Omega$ the conditions $\lambda>0, \mu<1$ are true, then there exists such a point $\left(\lambda^{\prime}, \mu^{\prime}\right) \in \Omega$ that $\lambda^{\prime}<\lambda, \mu^{\prime}>\mu$ and the value of the function

$$
g(\lambda, \mu)=\lambda-\frac{p^{B}}{t^{B}}(1-\lambda)(1-\mu)
$$

remained unchanged. This follows from the strict increase of this function with $\lambda, \mu$ and respect to condition (36), which together with (13) defines the set $\Omega$. Moreover, the value of the function $\sigma^{B}(\lambda, \mu)$ decreases according to the obtained expression (37), which contradicts the minimality of the criterion $\sigma^{B}(\lambda, \mu)$ on the set $\Omega$. It follows that the conditions $\lambda=0$ or $\mu=1$ are the necessary condition for the minimality of $\sigma^{B}(\lambda, \mu)$.

Theorem 2 allows us to find all the minimum points of the criterion $\sigma^{B}(\lambda, \mu)$ on the set $\Omega$ in case (25).

\subsubsection{Conditions for $x$, Under Which $\lambda=0$}

Let's Find the Conditions for $x$, Under Which $\lambda=0$, i.e. $\sigma^{B}(\lambda, \mu)$ Reaches an Absolute Minimum. Substituting in (31), Taking into Account the condition (25), We'Ll Obtain the Inequality

$$
x \leq \frac{P^{B}}{t^{B}}+\frac{t^{A}}{P^{A}}-\frac{P^{B}}{t^{B}}(1-\mu)=\frac{t^{A}}{P^{A}}+\frac{P^{B}}{t^{B}} \mu .
$$

On the right side of (38) is an increasing function from $\mu \in[0,1]$. Substituting the extreme values, we'll obtain the boundaries of a possible change of $x$

$$
\frac{t^{A}}{P^{A}} \leq x \leq \frac{t^{A}}{P^{A}}+\frac{P^{B}}{t^{B}}
$$

Moreover, the $\mu$ value should be chosen as low as possible, taking into account the strategy of necessary sufficiency of the $A$ player's defense. Therefore, (38) is satisfied as an equality, whence we'll obtain a solution of the $B$ player's minimum sufficiency

$$
\lambda=0, \mu=\frac{t^{B}}{P^{B}}\left(x-\frac{t^{A}}{P^{A}}\right) .
$$

Note that when the range (39) runs through, the $\mu$ value changes from 0 to 1 . Thus, the following lemma is proved.

Lemma 1. Let's suppose that conditions (14), (22) are satisfied. Then a necessary and sufficient condition for the point (40) to be a solution of the $B$ player's minimum sufficiency is condition (39).

Example 1. Let's suppose that there are $n=1$ directions with $T=1$ so that $t=p r$ and $Q=q, P=p$. Let
$r^{B}=1, r^{A}=2, x=2,5$. It is required to construct a Pareto set of parameters $(\lambda, \mu)$.

Solution. Condition (39)

$$
\frac{t^{A}}{P^{A}}=r^{A}=2 \leq x=2,5 \leq \frac{t^{A}}{P^{A}}+\frac{P^{B}}{t^{B}}=r^{A}+\frac{1}{r^{B}}=3 .
$$

is satisfy. Consequently, the permissible values of the parameter vector $(\lambda, \mu)$ is satisfy inequality (36) where only the right one is nontrivial, which takes the form

$x=2,5 \leq \frac{P^{B}}{t^{B}}+\lambda+\frac{t^{A}}{P^{A}}-\frac{P^{B}}{t^{B}}(1-\lambda)(1-\mu)=3+\lambda-(1-\lambda)(1-\mu)$.

The latter is equivalent to the inequality

$$
0,5+\lambda \geq(1-\lambda)(1-\mu)=1-\lambda-\mu+\lambda \mu .
$$

The equality holds on the Pareto border, whence, taking into account conditions (13), we'll obtain its parametric notation in the form

$$
\lambda=\frac{0,5-\mu}{2-\mu}, 0 \leq \mu \leq 0,5 .
$$

Note that the point (40)

$$
\lambda=0, \mu=\frac{t^{B}}{P^{B}}\left(x-\frac{t^{A}}{P^{A}}\right)=0,5
$$

represents its extreme point corresponding to the strategy of minimum sufficiency of the $B$ side defense, i.e. providing a minimum value of $\sigma^{B}$ or a minimum value $\lambda=0$, which can be verified by substituting the value $\mu=0,5$ obtained by formula (40) into the found parametric representation of the Pareto boundary.

The set $\Omega$ and its Pareto boundary $\Omega^{\prime}$ are shown in the Figure 1.

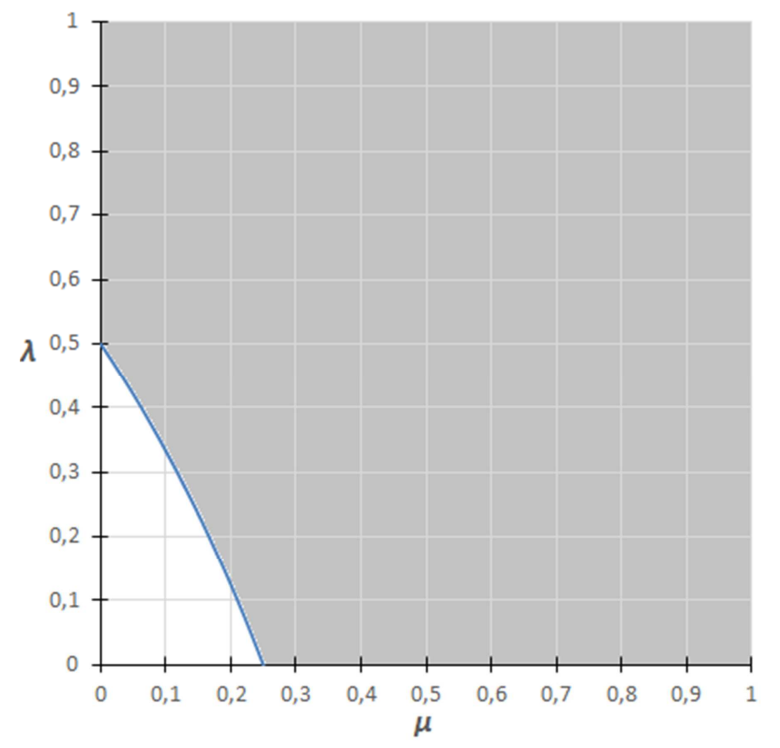

Figure 1. The set $\Omega$ and its Pareto boundary $\Omega^{\prime}$ in example 1. 


\subsubsection{The Case When}

$$
x>\frac{t^{A}}{p^{A}}+\frac{p^{B}}{t^{B}}>\frac{p^{B}}{t^{B}}+\lambda .
$$

Then the left inequality in (36) is satisfy, and the right inequality for minimal equilibria on $\sigma^{B}$ is satisfy as equality

$$
x=\frac{P^{B}}{t^{B}}+\lambda+\frac{t^{A}}{P^{A}}-\frac{P^{B}}{t^{B}}(1-\lambda)(1-\mu) .
$$

This follows from condition (30) of the strict increase of the function $\sigma^{B}$ on $\lambda, \mu$, which in this case is satisfied by virtue of (41).

Since $\lambda=1$ is impossible due to the conditions of the previous paragraph, then the minimal solution on $\sigma^{B}$ is obtained when $\mu=1$, i.e. when

$$
x=\lambda+\frac{t^{A}}{P^{A}},
$$

where we get

$$
\lambda=x-\frac{t^{A}}{P^{A}} .
$$

Moreover, it follows from (41) that

$$
\lambda=x-\frac{t^{A}}{P^{A}}>\frac{P^{B}}{t^{B}}>0,
$$

The condition $\lambda \leq 1$, is remains which by virtue of (42), is equivalent to the inequality

$$
x \leq \frac{t^{A}}{P^{A}}+1 .
$$

Combining (41) and (43), we'll obtain the boundaries of a possible change of $x$

$$
\frac{t^{A}}{P^{A}}+\frac{P^{B}}{t^{B}}<x \leq \frac{t^{A}}{P^{A}}+1 .
$$

Moreover, the minimal solution on $\sigma^{B}$ has the form

$$
\lambda=x-\frac{t^{A}}{P^{A}}, \mu=1 .
$$

Note that when $x$ runs through the range (44) the $\lambda$ value changes from $\frac{P^{B}}{t^{B}}$ to 1 . Thus, the following lemma is proved.

Lemma 2. Let's suppose that conditions (14), (41) are satisfied. Then a necessary and sufficient condition for the point (45) to be a solution of the $B$ player's minimum sufficiency is condition (44).

\subsection{LR and RL Options}

Lemma 3. Under condition (15) and

$$
x \geq \frac{t^{A}}{P^{A}} \cdot \frac{1}{1-\lambda}, \lambda \in[0,1),
$$

there is only one minimal equilibrium on $\sigma^{B}$

$$
\sigma^{B}=\lambda, \sigma^{A}=1
$$

Proof. In fact, in the LP option we'll get

$$
\sigma^{B}=\lambda, \sigma^{A}=1
$$

under conditions

$$
1-\frac{t^{B}}{P^{B}} x \leq \sigma^{A}=1 \leq 1,0 \leq \sigma^{B}=\lambda \leq 1-\frac{t^{A}}{P^{A}} \cdot \frac{1}{x},
$$

from which only the right inequality in the right double inequality is nontrivial, which is equivalent to the condition $x \geq \frac{t^{A}}{P^{A}} \cdot \frac{1}{1-\lambda}$.

Remark 5. When

$$
x \leq \frac{P^{B}}{t^{B}}(1-\mu), \mu \in[0,1),
$$

there is only one minimal equilibrium $\sigma^{B}=1, \sigma^{A}=\mu$. on $\sigma^{A}$. This follows from symmetry by virtue of Remark 2 .

\subsection{RR Option}

In this case equilibria do not exist. This can be seen directly from the general parametric representation of equilibria (12) - (14). In fact, in this case the restrictions has the form

$$
0 \leq \sigma^{A}=1<1-\frac{t^{B}}{P^{B}} x, 0 \leq \sigma^{B}=1<1-\frac{t^{A}}{P^{A}} \cdot \frac{1}{x}
$$

and obviously incompatible.

\subsection{Comparison of Minimum Equilibria on $\sigma^{B}$}

\subsubsection{Minimum Equilibria on $\sigma^{B}$}

Under Condition (39), the Minimum Equilibria on $\sigma^{B}$ Obtained in Lemmas 1 and 3 Are Comparable. By Lemma 1, the Solution of the Necessary Sufficiency of Defense for the Side $B$ is (40)

$$
\lambda=0, \mu=\frac{t^{B}}{P^{B}}\left(x-\frac{t^{A}}{P^{A}}\right) .
$$

By the formula (28) we'll obtain 


$$
\sigma^{B}=1-\frac{x-\frac{P^{B}}{t^{B}}-\lambda}{x g(\lambda, \mu)}
$$

where according to (3.18)

$$
g(\lambda, \mu)=1-\frac{P^{B}}{t^{B}} \cdot \frac{P^{A}}{t^{A}}(1-\mu)(1-\lambda)
$$

Substituting the expression for $\mu$ from (48) into (50) when $\lambda=0$, we'll obtain

$$
g(\lambda, \mu)=1-\frac{p^{B}}{t^{B}} \cdot \frac{p^{A}}{t^{A}}\left(1-\frac{t^{B}}{p^{B}}\left(x-\frac{t^{A}}{p^{A}}\right)\right)=\frac{p^{A}}{t^{A}}\left(x-\frac{p^{B}}{t^{B}}\right) .
$$

Whence, by virtue of (49) when $\lambda=0$, we'll obtain

$$
\sigma^{B}=1-\frac{t^{A}}{x P^{A}}
$$

Now, by formula (32), we have

$$
\sigma^{A}=(1-\mu) x \frac{P^{A}}{t^{A}} \cdot \frac{x-\frac{P^{B}}{t^{B}}}{x g(\lambda, \mu)}+\mu=(1-\mu) x \frac{P^{A}}{t^{A}} \cdot \frac{t^{A}}{x P^{A}}+\mu=1 \text {. }
$$
(39)

There is the condition (46) by Lemma 3 under condition

$$
x \geq \frac{t^{A}}{P^{A}} \cdot \frac{1}{1-\lambda}, \lambda \in[0,1)
$$

when

$$
\lambda=1-\frac{t^{A}}{x P^{A}} .
$$

Therefore, there is a solution of the necessary sufficiency of defense for the $B$ party (47)

$$
\sigma^{B}=\lambda, \sigma^{A}=1,
$$

which coincides with (52), (53) taken into account (55).

Thus, the LL and LR options provide the same solution.

\subsubsection{Dominates the Solution}

Under Condition (44), the Solutions Obtained in Lemmas 2 and 3 Are Comparable. By Lemma 2, the Solution Is (45)

$$
\lambda=x-\frac{t^{A}}{P^{A}}, \mu=1 .
$$

By the formula (49) we'll obtain

$$
\sigma^{B}=1-\frac{x-\frac{P^{B}}{t^{B}}-\lambda}{x g(\lambda, \mu)}=1-\frac{\frac{t^{A}}{P^{A}}-\frac{P^{B}}{t^{B}}}{x},
$$

since, according to (50) $g(\lambda, \mu)=1$ when $\mu=1$.

Moreover, according to (53) when $\mu=1$. we'll obtain

$$
\sigma^{A}=1 .
$$

By Lemma 3, there exists a solution (55), (56)

$$
\sigma^{B}=1-\frac{t^{A}}{x P^{A}}, \sigma^{A}=1,
$$

which dominates the solution (58), (59).

Thus, the LR option provides a better solution than LL option.

\subsubsection{Case When There Are no Solutions}

When

$$
x>\frac{t^{A}}{p^{A}}+1,
$$

in the LL option, and in the LR option there is a solution (59). From $4.1-4.3$ it follows that the following theorem is correct.

Theorem 3. In the case (26)

$$
x>\frac{t^{A}}{P^{A}},
$$

the LR option provides the solution of the necessary defense sufficiency for the $B$ party (59) no worse than the solutions of the LL option.

The classification of non-dominant equilibria of minimum defense sufficiency for the $B$ party is presented in the table 1 .

Table 1. Classification of non-dominated solutions $\sigma^{B}$ of the minimum defense sufficiency for the $B$ party when $t^{B} \geq P^{B}, t^{A} \geq P^{A}$ and $\sigma^{A}=(1-\mu) P^{A} / t^{A} x\left(1-\sigma^{B}\right)+\mu, g(\lambda, \mu)=1-\frac{P^{B}}{t^{B}} \cdot \frac{P^{A}}{t^{A}}(1-\mu)(1-\lambda)$.

\begin{tabular}{llll}
\hline $\mathbf{N}$ & Range $x=Y^{B} / Y^{A}$ & Options $(\lambda, \mu)$ & Share of $\sigma^{B}$ \\
\hline 1 & {$\left[P^{B} / t^{B}, t^{A} / P^{A}\right]$} & $(0,0)$ & $\frac{P^{B} / t^{B}\left(1-x P^{A} / t^{A}\right)}{x\left(1-P^{B} / t^{B} \cdot P^{A} / t^{A}\right)}$. \\
2 & {$\left[t^{A} / P^{A}, t^{A} / P^{A}+P^{B} / t^{B}\right]$} & $\left(0, P^{B} / t^{B}\left(1-t^{A} / P^{A}\right)\right)$ & $1-\left(x-P^{B} / t^{B}-\lambda\right) /(x g(\lambda, \mu))$ \\
3 & {$\left[t^{A} / P^{A}+P^{B} / t^{B}, t^{A} / P^{A}+1\right]$} & $\left(x-t^{A} / P^{A}, 1\right)$ & $1-\left(t^{A} / P^{A}-P^{B} / t^{B}\right) / x$ \\
4 & {$\left[t^{A} / P^{A}, \infty\right)$} & $\left(x-t^{A} / P^{A}, 1\right)$ & $1-t^{A} /\left(x P^{A}\right)$ \\
\hline
\end{tabular}


Remark 6. Solutions 1 and 3 represent a special case of solution 2 . We remain the solution 3 , although it is dominated by solution 4, since cases 1-3 correspond to the most interesting case of LL, when the Pareto boundary $\Omega^{\prime}$ of the set $\Omega$ is different from $\{(1,1)\}$.

Remark 7. For non-dominated equilibria of minimal sufficiency for the $A$ party the solutions are obtained from symmetry when the indices of the parties are replaced by opposite ones.

Remark 8. The obtained formulas for $\sigma^{B}$ and $\sigma^{A}$ can be used in the dynamic extension of the model according to the scheme [13], in which the equations of system's motion will have the form:

$$
Y_{k+1}^{A}=\max \left\{Q^{B} Y_{k}^{A}\left(1-\sigma_{k}^{A}\right), Y_{k}^{A}\left(1-\sigma_{k}^{A}\right)-t^{B} Y_{k}^{B} \sigma_{k}^{B}\right\}+Y_{k}^{A} \sigma_{k}^{A}
$$

and

$Y_{k+1}^{B}=\max \left\{Q^{A} Y_{k}^{B}\left(1-\sigma_{k}^{B}\right), Y_{k}^{B}\left(1-\sigma_{k}^{B}\right)-t^{A} Y_{k}^{A} \sigma_{k}^{A}\right\}+Y_{k}^{B} \sigma_{k}^{B}$,

where

$$
x_{k}=Y_{k}^{B} / Y_{k}^{A}, k=0,1, \ldots
$$

Remark 9. The question is, what solutions remain when condition (15) $P^{B} / t^{B}<1, P^{A} / t^{A}<1$ is not satisfied?

The solution $(0,0)$ in the parametric form on the first interval $\left[P^{B} / t^{B}, t^{A} / P^{A}\right]$ remain if $P^{B} / t^{B} \leq P^{A} / t^{A}$ and $P^{B} / t^{B} \cdot P^{A} / t^{A}<1$.

For example, $\quad P^{B} / t^{B}=1,5 ; P^{A} / t^{A}=0,5$. Then $P^{B} / t^{B}=1,5 \leq t^{A} / P^{A}=2$ and $P^{B} / t^{B} \cdot P^{A} / t^{A}=0,75<1$.

The solution on the remaining half-interval $x \geq P^{A} / t^{A}$ has the usual form $\left(1-t^{A} /\left(x P^{A}\right), 1\right)$ in a parametric form, which allows us to build a dynamic extension of the model according to the previous scheme.

\subsection{Numerical Example}

Example 2. Let's suppose that there are $n=2$ symmetric directions with $T=1$ so that $t=\frac{p r}{2}$ and let

$$
\begin{aligned}
& Y^{A}=70 ; p^{B}=0,7, r^{B}=2, Q^{A}=q^{A}=0,3 ; \\
& Y^{B}=100 ; p^{A}=0,8, r^{A}=4, Q^{B}=q^{B}=0,2 .
\end{aligned}
$$

It is required to find the minimum equilibrium on $\sigma^{B}$.

Solution. With this data $t^{B}=\frac{p^{B} r^{B}}{2}=0,7 ; t^{A}=\frac{p^{A} r^{A}}{2}=1,6$. Let's find the aspect ratio $x=100: 70=1,43$. The condition (25)

$$
\frac{P^{B}}{t^{B}}=1 \leq x \leq \frac{t^{A}}{P^{A}}=2
$$

is satisfied and the only non-dominated equilibrium is obtained by the formulas (23), (24)

$$
\sigma^{B}=\frac{P^{B}}{t^{B}} \frac{\left(1-\frac{P^{A}}{t^{A}} x\right)}{x\left(1-\frac{P^{B}}{t^{B}} \cdot \frac{P^{A}}{t^{A}}\right)} \approx 0,40,
$$

and

$$
\sigma^{A}=\frac{P^{A}}{t^{A}} x\left(1-\sigma^{B}\right) \approx 0,43
$$

Thus, for the side $B$ it is enough to allocate $40 \%$ of its forces to defense, and for the side $A$ it is enough to allocate $43 \%$ of its forces to defense.

\section{Conclusion}

In this work, we proposed the symmetrization of the "attack-defense" model defined and studied by Germeyer. In the military models points are usually interpreted as directions and characterizes the spatial distribution of defense resources across the width. It is also possible to distribute the resources in depth in relation with the separation of the defense. The parties' resources are generally heterogeneous. All these areas of generalization of the classical "attack-defense" model were studied by the authors in previous works. In reality, there is also a symmetry of the conflict, when both sides attack and defend at the same time. Therefore, in the present work, a symmetric extension of the model was proposed, in which the parties simultaneously participate in two games, notably in one game the each side is an attack party and in the other game the each side is a defense party. The solution in the resulting doubled game is defined as the non-dominated Nash's equilibrium by Pareto. A classification of such equilibria is given depending on the balance of parties' forces. The extreme points of the Pareto' sets corresponding to the minimum of the share of the stronger side directed to defense are distinguished. Or the same thing, the extreme points of the Pareto' sets corresponding to the maximum share of the stronger side directed to the attack, which makes sense when planning a defense's breakthrough of the weaker side.

\section{References}

[1] Perevozchikov A. G., Lesik I. A., Shapovalov T. G. Multilevel generalization of the "attack-defense" model // Bulletin of TSU. Seria Applied Mathematic. 2017, No. 1, p. 39-51.

[2] Reshetov V. Y., Perevozchikov A. G., Lesik I. A. A Model of Overpowering a Multilevel Defense System by Attack // Computational Mathematics and Modeling, 2016, Vol. 27, No. 2, p. 254-269. 
[3] Reshetov V. Y., Ptrevozchikov A. G., Lesik I. A. Multi-Level Defense System Models: Overcoming by Means of Attacks with Several Phase Constraints // Moscow University Computational Mathematics and Cybernetics, 2017, Vol. 1, No. 1, p. 25-31.

[4] Reshetov V. Y., Perevozchikov A. G., Yanochkin I. E. An Attack-Defense Model with Inhomogeneous Resources of the Opponents // Journal of Computational Mathematics and Mathematical Physics, 2018, Vol. 58, No. 1, p. 38-47.

[5] Germeier Y. B. Introduction to the theory of operations research. Moscow, Science, 1971.

[6] Karlin S. Mathematical methods in game theory, programming and economics. Moscow, Mir, 1964.

[7] Ogaryshev V.F. Mixed strategies in one generalization of the Gross' problem // Journal of Computational Mathematics and Mathematical Physics. 1973. Vol. 13. No. 1. p. 59-70.

[8] Molodtsov D. A. Gross' model in case of conflicting interests // // Journal of Computational Mathematics and Mathematical Physics. 1972. Vol. 12, No. 2, p. 309-320.
[9] Danilchenko T. N., Masevich K. K. Multistage game of two persons with a "cautious" second player and consistent transmission of information // Journal of Computational Mathematics and Mathematical Physics, 1974. Vol. 19. No. 5. pp. 1323-1327.

[10] Krutov B. P. Dynamic quasi-informational extensions of games with an expandable coalition structure. Moscow, $\mathrm{CC}$ of RAS, 1986.

[11] Hohzaki R., Tanaka $V$. The effects of players recognition about the acquisition of his information by his opponent in an attrition game on a network // In Abstract of 27th European conference on Operation Research 12-15 July 2015 University of Strathclyde. - EURO2015.

[12] Vasin A. A., Morozov V. V. Game theory and models of mathematical economics. Moscow, MAX Press, 2005.

[13] Lesik A. I., Perevozchikov A. G., Reshetov V. Y. A multi-step generalization of the "attack-defense" model // Bulletin of TSU. Seria Applied Mathematic. 2017, No. 2, p. 99-110. 